\title{
Tube Formation in Reverse Silica Gardens
}

\author{
Jason J. Pagano, Tamás Bánsági, Jr., and Oliver Steinbock* \\ Department of Chemistry and Biochemistry, Florida State University, Tallahassee, Florida 32306-4390
}

Received: February 23, 2007; In Final Form: April 3, 2007

\begin{abstract}
The flow injection of sodium silicate solution into a large reservoir of lighter cupric sulfate solution creates single, downward growing precipitation tubes. These hollow structures have diameters in the range of $0.8-$ $2.4 \mathrm{~mm}$ and can grow several centimeters in length. Four distinct growth regimes are observed, and their stability in terms of flow rate and cupric sulfate concentration is investigated. Three of these growth regimes resemble behavior reported earlier for the injection of cupric sulfate into silicate solution. However the "reverse" conditions studied here reveal one distinctly different regime in which tube growth is limited by repetitive fracturing. The lengths of the broken-off tube segments and the times between subsequent break-off events can be described by $\log -$ normal distributions.
\end{abstract}

\section{Introduction}

Self-organizing reaction-precipitation processes are ubiquitous in nature. They can cause the formation of permanent tubular structures due to the interplay of chemical processes and transport phenomena such as diffusion and fluid flow. The spectrum of examples is extremely diverse and includes hollow micrometer-scale fibers in cement, ${ }^{1-3}$ biomineralized shells of marine algae ${ }^{4,5}$ precipitation tubes in corrosion systems, ${ }^{6-11}$ mineral deposits in caverns ${ }^{12-15}$ (e.g., soda straws), and so-called "black smokers" which are up to $30 \mathrm{~m}$ tall tower-like hydrothermal vents on the ocean floor. ${ }^{16}$

Among the tube-forming reaction-precipitation systems, the most widely known example is a class of reactions known as "silica gardens" or "chemical gardens". ${ }^{17-25}$ These reactions create millimeter-scale precipitation tubes that grow upward at rates of millimeters per second to millimeters per day. Typically, the process is initiated by seeding small salt crystals into a solution of sodium silicate (waterglass). The subsequent tube growth involves colloidal intermediates and is driven by the coprecipitation of amorphous silica and metal hydroxides (or oxides). A great variety of salts can be employed as seed crystals excluding those of group 1A elements. Moreover, hollow fibers can also form if waterglass is replaced by solutions containing anions such as borate or carbonate.

Silica gardens have been studied since the 17 th century. ${ }^{26,27}$ Many of these early studies were motivated by the amazing "life-like vegetation" created during the precipitation processes, and even in the 19th century some researchers considered silica gardens to be pre-biotic life forms and models for the genesis of life. ${ }^{28}$ Obviously, advances in biochemistry have dismissed these ideas. Nonetheless, silica gardens are still familiar to today's general public partly because the phenomenon is discussed in the popular literature ${ }^{29-31}$ and also because the needed reactants are included in many educational chemistry kits. Perhaps more surprisingly, the study of silica gardens has experienced a renaissance in modern research and many of their intriguing chemical and physical aspects have been discovered only in very recent years. For instance, transmission electron

* To whom correspondence should be addressed. Fax: 01850644 8281. Tel: 01850644 4824. E-mail:steinbck@chem.fsu.edu. microscopy (TEM) studies by Collins et al. showed that precipitation tubes in the aluminum silicate system are hierarchical nanostructures. ${ }^{32}$ Other experiments revealed that the chemically activated tube walls are highly efficient Brønsted acid catalysts $^{33,34}$ and that chiral tubes can form in the presence of strong magnetic fields. ${ }^{35}$ Tube growth was also investigated under microgravity conditions. ${ }^{36-38}$

The qualitative description of tube growth in silica gardens involves the formation of a semipermeable, colloidal membrane around the dissolving seed crystal. ${ }^{39}$ As the concentration of the metal salt ion increases, a buildup of osmotic pressure occurs and the inflow of water across the membrane causes it to breach. This breach allows the release of buoyant solution into the surrounding waterglass, and tube growth occurs around a vertical jet due to the build-up of solid products. Tube growth in the silica gardens is also closely related to the setting of the cement which is essentially a mixture of limestone and clay (tricalcium silicate, dicalcium silicate, tricalcium aluminate). As water is added to this mixture, silicate grains become surrounded by a colloidal membrane from which micrometer-scale tubes emerge. This process is obviously similar to the one in silica gardens, but in cement, the reactants are arranged in a reverse fashion and silicate particles are the actual seeds. Although in recent years some studies have been conducted to elucidate this rather complex hydration mechanism, many of the underlying phenomena remain unexplained. ${ }^{40}$

A few of the difficulties hampering quantitative studies of silica gardens (and the setting of cement) are the continuous dissolution of the seed particle, the blistering of the membrane at multiple sites, the presence of air bubbles, ${ }^{41}$ and the interaction of neighboring tubes. To eliminate these problems, ThouvenelRomans et al. developed a method which allows the collection of quantitative data based on flow-controlled injection. ${ }^{42-45}$ This method replaces the seed particle with a "seed solution" which is injected into a large volume of waterglass at constant flow rates of several milliliters per hour. This approach yields long, solitary tubes that grow in upward direction and has allowed the identification of three distinct growth regimes coined jetting, popping, and budding. We note that these growth regimes depend strongly on the density difference between waterglass in the reservoir and the injected seed solution. 
All earlier investigations on flow-controlled tube growth involved the injection of salt solutions into waterglass. In this article, however, we analyze tube formation under reverse conditions, that is, conditions in which waterglass is injected into a large reservoir of cupric sulfate solution. Furthermore, tubes are growing downward because the waterglass is denser than the employed cupric sulfate solutions. In particular, we report the existence of reverse jetting, popping, and budding regimes and, more importantly, describe a qualitatively novel regime in which growth dynamics are affected by the repetitive break-off of large tube segments.

\section{Experimental Section}

Solutions of $1.0 \mathrm{M}$ sodium silicate $\left(\mathrm{Na}_{2} \mathrm{SiO}_{3} \cdot 5 \mathrm{H}_{2} \mathrm{O}\right.$, Fisher $)$ are prepared and then injected into a large reservoir containing cupric sulfate solution $\left(\mathrm{CuSO}_{4}\right.$, Aldrich). The injection is carried out in a downward direction through a vertical glass capillary (length $=6 \mathrm{~cm}$; inner diameter $\approx 1 \mathrm{~mm}$ ). The injection rate is controlled and predetermined using a syringe pump (KD Scientific 200). A cylindrical glass vessel (height $\approx 25 \mathrm{~cm}$; inner diameter $=3.5 \mathrm{~cm}$ ) serves as the container for the cupric sulfate solution. Solutions are prepared in nanopure water $(18 \mathrm{M} \Omega \mathrm{cm}$, Easy-pure UV, Barnstead). All experiments are carried out at a temperature of $(21 \pm 1){ }^{\circ} \mathrm{C}$.

At constant temperature, the density of the cupric sulfate solution has an essentially linear dependence on cupric sulfate concentration. Consequently, the density difference $\Delta \rho$ between the sodium silicate solution (waterglass) and the cupric sulfate solutions obeys a linear relation $\Delta \rho=\rho_{\mathrm{WG}}-\left(\rho_{\mathrm{H}_{2} \mathrm{O}}+\xi\left[\mathrm{CuSO}_{4}\right]\right)$ where the density of waterglass and the density of water equal $\rho_{\mathrm{WG}}=1124 \mathrm{~kg} / \mathrm{m}^{3}$ and $\rho_{\mathrm{H}_{2} \mathrm{O}}=998 \mathrm{~kg} / \mathrm{m}^{3}$, respectively. The proportionality constant $\xi$ has been measured as $0.15 \mathrm{~kg} / \mathrm{mol}{ }^{46}$

Optical micrographs of the growth dynamics are acquired with a monochrome charged-coupled device camera (COHU 2122) connected to a PC via a frame grabber board (Data Translation DT3155, $640 \times 480$ pixels at 8 bit/pixel). Image sequences are captured using HL Image ++97 software. The images are collected at a typical rate of 1-2 frame/s and then analyzed using MATLAB routines.

\section{Results}

In typical silica gardens, tube growth occurs in an upward direction from a seed crystal at the bottom of a waterglassfilled reaction vessel. As described in the Introduction section, this article focuses on the reverse conditions, and all precipitation structures are growing in the downward direction. Consequently, no vertical structures will form from a seed located at the bottom of the vessel but one rather observes the formation of a bluish, gel-like mound with no clear macroscopic structure. Nonetheless, tubes formed under reverse conditions can be studied if a sodium silicate particle is held mechanically in the upper portion of a salt solution such as aqueous cupric sulfate. Figure 1 gives a typical example for tube growth under such conditions. In this experiment, the sodium silicate crystal is glued to a thin glass capillary. Upon submerging the seed into the copper solution, the crystal is rapidly covered with a blue, colloidal substance (Figure 1a) that is likely to contain large amounts of colloidal copper hydroxide. Subsequently, we observe the formation of a downward growing tube as well as a pile of bluish product at the bottom of the reaction container (parts $b$ and $c$ of Figure 1). Close inspection of our image data suggests that the tube grows around a descending fluid jet which can be vaguely discerned in Figure 1b. Eventually, tube and

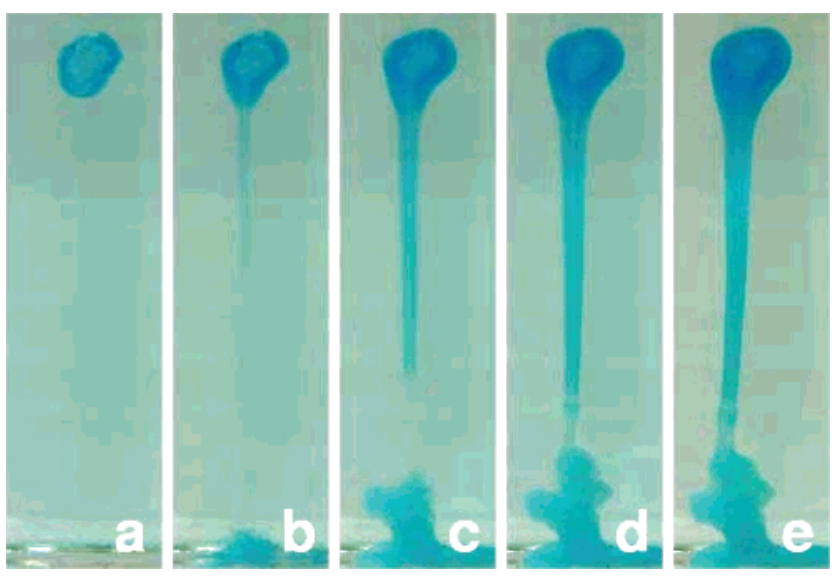

Figure 1. Image sequence of a downward growing tube. The reactants are a single sodium silicate particle suspended in a $0.075 \mathrm{M} \mathrm{CuSO}_{4}$ solution. The particle is glued to a glass rod to prevent it from sinking to the bottom of the reaction vessel. The field of view is $(2.1 \times 7.3)$ $\mathrm{cm}^{2}$. The time elapsed between snapshots is $1.0 \mathrm{~min}$.

product pile meet (parts $\mathrm{d}$ and e of Figure 1) and all further product formation simply increases the size of the "stalagmitelike" pile.

Although the experiment in Figure 1 demonstrates that tube growth can occur from a silicate seed submerged in cupric sulfate solution, the setup does not allow satisfactory control of the experimental conditions because the size and the structure of the seed as well as the glued contact are difficult to reproduce. Moreover, the silicate seed is of finite mass and undergoes continuous dissolution which introduces undesired transients that are hard to characterize and/or control. In the following, we therefore employ the injection method described in the Experimental Section. Using this technique, we are able to distinguish four qualitatively different growth regimes.

A representative example for each of these four regimes is shown in the image sequences of Figure 2. The first row (a) illustrates tube formation in a dynamic regime that will be referred to as reverse jetting since it is reminiscent of the jetting growth described in two earlier studies that involved the injection of cupric sulfate into a large volume of waterglass. Reverse jetting growth occurs for high flow rates $(35-200 \mathrm{~mL} /$ h) and requires high density differences (100-120 kg/m ${ }^{3}$ ) between the cupric sulfate solution in the reservoir and the denser, injected sodium silicate solution. Specifically, we find that solitary tubes form around a continuous, descending jet. Due to differences in refractive index, this jet can be seen in Figure $2 \mathrm{a}$ as a very faint line. The smooth and cylindrical precipitation tube appears only slightly darker than the descending fluid jet while the conical base close to the injection nozzle is rather thick and readily discernible. To guide the reader, we mark the approximate extension of the tube in the last snapshot by a black arrow. For the parameters of this specific experiment, the growth velocity of the tube and the average, outer tube radius are $3.8 \mathrm{~mm} / \mathrm{s}$ and $750 \mu \mathrm{m}$, respectively. The tubes grown in the reverse jetting regime are rather delicate, and we did not succeed in extracting them for subsequent processing or analysis.

Reverse jetting behavior ceases and gives way to a qualitative different form of tube growth if the density difference between injected and outer solution is smaller than $110 \mathrm{~kg} / \mathrm{m}^{3}$ and the flow rate is lowered to values between approximately 25 and $185 \mathrm{~mL} / \mathrm{h}$. Figure $2 \mathrm{~b}$ shows a sequence of eight consecutive snapshots that illustrate the dynamics of this reverse popping growth. Most importantly, it involves the periodic release of drop-like pieces of colloidal matter which sink to the bottom 


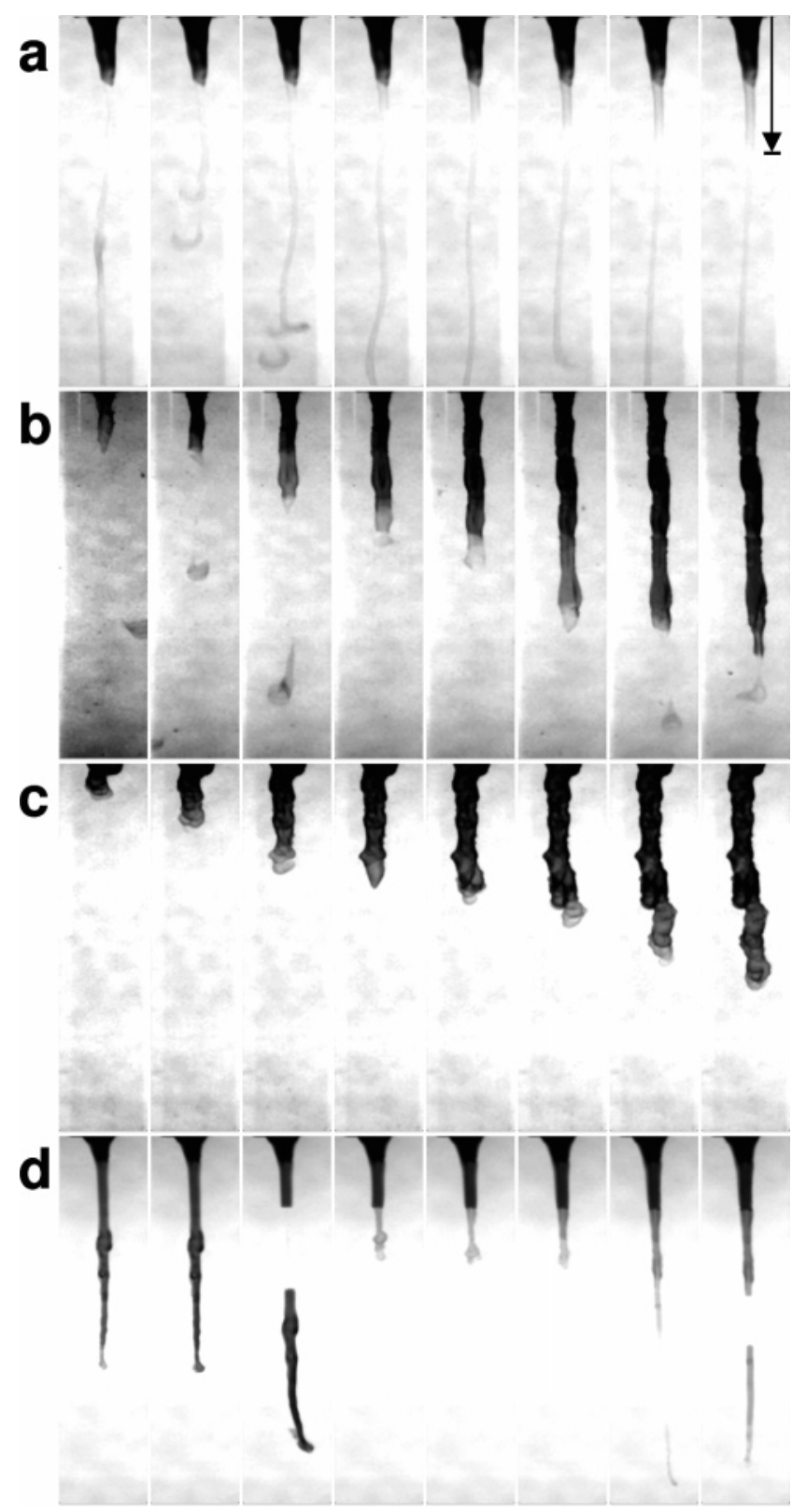

Figure 2. Image sequences illustrating four reverse growth regimes observed for the injection of sodium silicate $(1.0 \mathrm{M})$ into cupric sulfate solution. The growth regimes are referred to as (a) jetting, (b) popping, (c) budding, and (d) fracturing. Flow rates, cupric sulfate concentrations, and density differences are as follows: (a) $50.0 \mathrm{~mL} / \mathrm{h}, 0.107 \mathrm{M}$, $110 \mathrm{~kg} / \mathrm{m}^{3}$, (b) $30.0 \mathrm{~mL} / \mathrm{h}, 0.173 \mathrm{M}, 100 \mathrm{~kg} / \mathrm{m}^{3}$, (c) $5.0 \mathrm{~mL} / \mathrm{h}, 0.25 \mathrm{M}$, $90 \mathrm{~kg} / \mathrm{m}^{3}$, and (d) $1.1 \mathrm{~mL} / \mathrm{h}, 0.075 \mathrm{M}$, and $115 \mathrm{~kg} / \mathrm{m}^{3}$. The black arrow in the last snapshot of sequence (a) marks the extension of the actual tube. The times elapsed between snapshots are (a) $0.5 \mathrm{~s}$, (b) $12.5 \mathrm{~s}$, (c) $10.0 \mathrm{~s}$, and (d) $15.0 \mathrm{~s}$. All image areas are $(1.3 \times 5.4) \mathrm{cm}^{2}$.

of the container. For the specific parameters of the experiment in Figure 2b, we find an average oscillation period of $1.8 \mathrm{~s}$. The average speed of tube growth is $0.30 \mathrm{~mm} / \mathrm{s}$, and the average outer radius is $1.2 \mathrm{~mm}$. Moreover, we observe that the tubes formed in this oscillatory growth mode are wider and thicker than those synthesized under "non-reverse" jetting conditions.

The third growth regime, in the following called reverse budding, can be observed for even smaller density differences between the two reactant solutions. As illustrated in Figure 2c, reverse budding yields bulging, unbranched structures that perhaps are best described as a tube-like chain of hollow nodules. The growth dynamics are similar to that in the reverse popping regime but no detectable fragments of colloidal matter

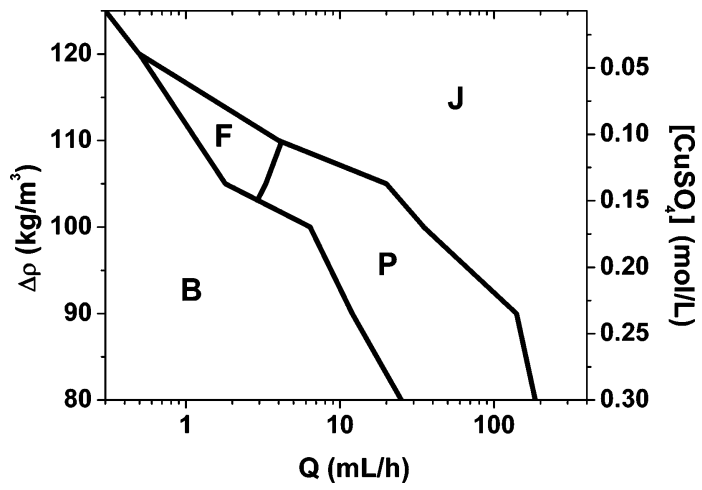

Figure 3. Phase diagram for reverse growth conditions. Shown are regions with the following: $(\mathrm{J})$ reverse jetting, $(\mathrm{P})$ reverse popping, (B) reverse budding, and (F) fracturing dynamics. The silicate concentration is kept constant at $1.0 \mathrm{M}$.

are released. The overall process involves the repetitive generation and expansion of small colloidal bubbles. Once these nodules reach a critical size, they burst and nucleate a new nodule thus completing the growth cycle.

The latter three regimes and the resulting macroscopic tube morphologies are similar to the jetting, popping, and budding growth observed earlier for non-reverse conditions. For reverse conditions, however, we succeeded in identifying a fourth distinct regime, which, for the lack of a better term, will be referred to as fracturing growth. The image sequence in Figure $2 \mathrm{~d}$ presents a typical example for this novel type of tube formation. During rather long periods, the tubular structures grow steadily downward although no jet of sodium silicate solution can be detected. The steady growth is interrupted by major break-off events, two of which are captured in the third and eighth snapshot of Figure 2d. During these catastrophic events, long, cylindrical segments split off and sink toward the bottom. The break-off events repeat rhythmically but lack a unique period. Also, the length of the released fragment can vary significantly.

To further characterize tube formation under reverse conditions, we survey the growth behavior by systematically varying experimental parameters. Three key parameters are the flow rate $Q$ and the reactant concentrations of $\mathrm{Na}_{2} \mathrm{SiO}_{3}$ and $\mathrm{CuSO}_{4}$. The two concentrations affect the reaction rates and determine the density difference $\Delta \rho$ between the reactant solutions in the reservoir and the injected jet as well as the viscosities of the fluids. We note that the nozzle geometry appears to be of no relevance as long as the nozzle diameter is not changed substantially and undesired backflow of the reservoir fluid into the injection channel is avoided. Moreover, the system temperature is not varied because we do not expect to obtain any qualitatively different information and, hence, no new insights from such experiments.

Figure 3 shows the phase diagram of reverse tube growth in terms of $\Delta \rho$ and $Q$. The density difference is varied by changing the initial cupric sulfate concentration while maintaining a constant silicate concentration in the injected jet. As described in the Experimental Section, the density difference depends linearly on cupric sulfate concentration. Notice that the flow rate is varied by more than 2 orders of magnitude from 0.3 to $200 \mathrm{~mL} / \mathrm{h}$.

The phase diagram in Figure 3 distinguishes between the four regimes described in the context of Figure 2. Each regime is bound to a unique region denoted by the first letter of its name. The assignment of an experiment to a specific regime is carried out by visual inspection of the growth dynamics. We note that 
a

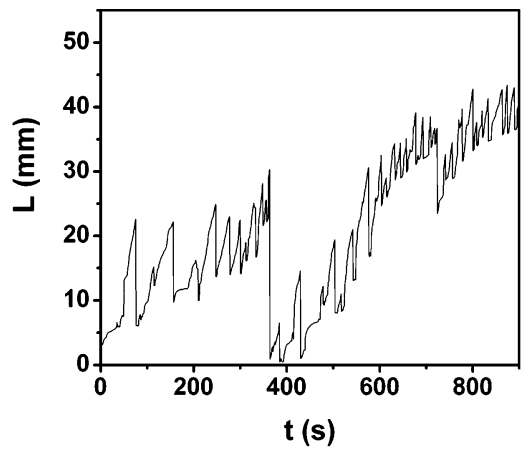

b

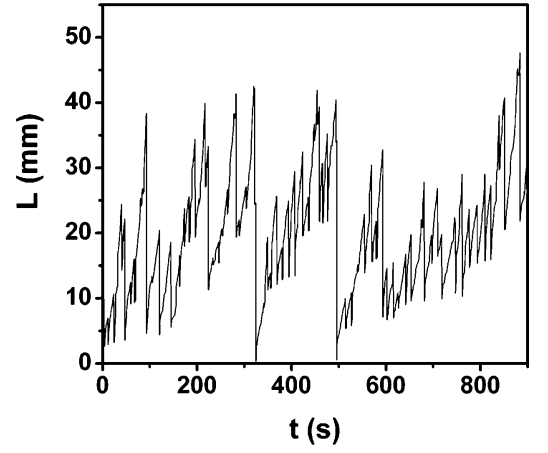

Figure 4. Representative examples illustrating the temporal evolution of the tube length $L$ under fracturing conditions. The two plots differ in flow rate only: (a) $Q=0.8 \mathrm{~mL} / \mathrm{h}$ and (b) $Q=1.4 \mathrm{~mL} / \mathrm{h}$. Other experimental parameters are as follows: $\left[\mathrm{CuSO}_{4}\right]=0.075 \mathrm{M}, \Delta \rho=$ $115 \mathrm{~kg} / \mathrm{m}^{3}$, and $\left[\mathrm{Na}_{2} \mathrm{SiO}_{3}\right]=1.0 \mathrm{M}$.

the assignment is not always unequivocal along the regime boundaries because of intermittent changes in dynamics. Nonetheless, our assignments aim to apply the following criteria: jetting must occur around a stable jet of solution and the tubes must have a nearly constant growth velocity; popping must involve the nearly periodic release of small colloidal droplets and a nearly constant increase in tube length with each popping event; budding is a rhythmic process which must not involve the release of any readily detectable colloidal or liquid substance; fracturing dynamics must involve the rhythmic release of well-formed tube segments of varying length. Using these definitions, we find that reverse jetting $(\mathrm{J})$ and budding (B) are the most dominant forms of tube growth in the parameter space investigated. Furthermore, for most but the smallest flow rates, these two regimes are separated by either oscillatory reverse popping $(\mathrm{P})$ or fracturing dynamics $(\mathrm{F})$. The transition between $(\mathrm{F})$ and $(\mathrm{P})$ occurs at a flow rate of approximately $3-$ $4 \mathrm{~mL} / \mathrm{h}$.

In the following, we provide data on fracturing in an attempt to better characterize this novel regime which appears to differ distinctly from all prior examples of tube formation in silica gardens and related experiments. Figure 4 shows two representative examples for the temporal evolution of the tube length $L$ under fracturing growth conditions. The length $L$ is defined as the tube's vertical extension from the lowest point of the injection nozzle downward. It is measured in an automated fashion from digital image sequences using in-house software. The experiments illustrated in parts a and $\mathrm{b}$ of Figure 4 differ only in the flow rate $Q$ which is $0.8 \mathrm{~mL} / \mathrm{h}$ in (a) and $1.4 \mathrm{~mL} / \mathrm{h}$ in (b). These values, along with the employed density difference of $\Delta \rho=115 \mathrm{~kg} / \mathrm{m}^{3}$, place the conditions well within the fracturing growth domain (cf. Figure 3). The two time traces in Figure 4 are very jagged and seemingly erratic. Each sudden decrease in $L$ corresponds to the break-off of a portion of the lower tube segment while the slower phases of increasing $L$

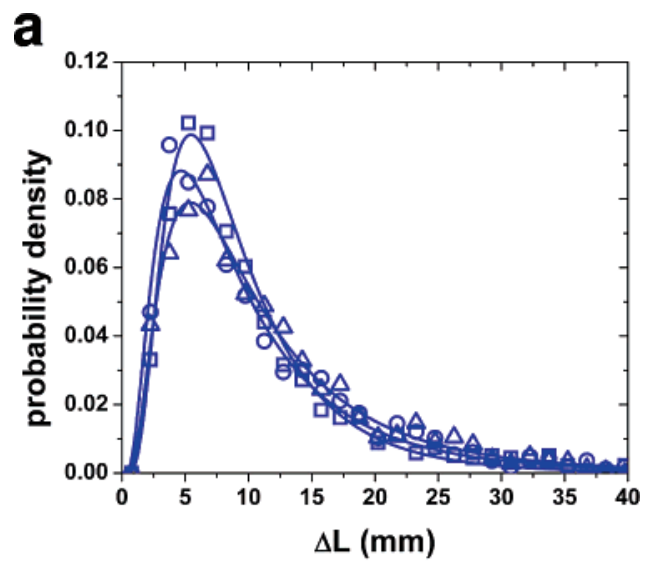

b
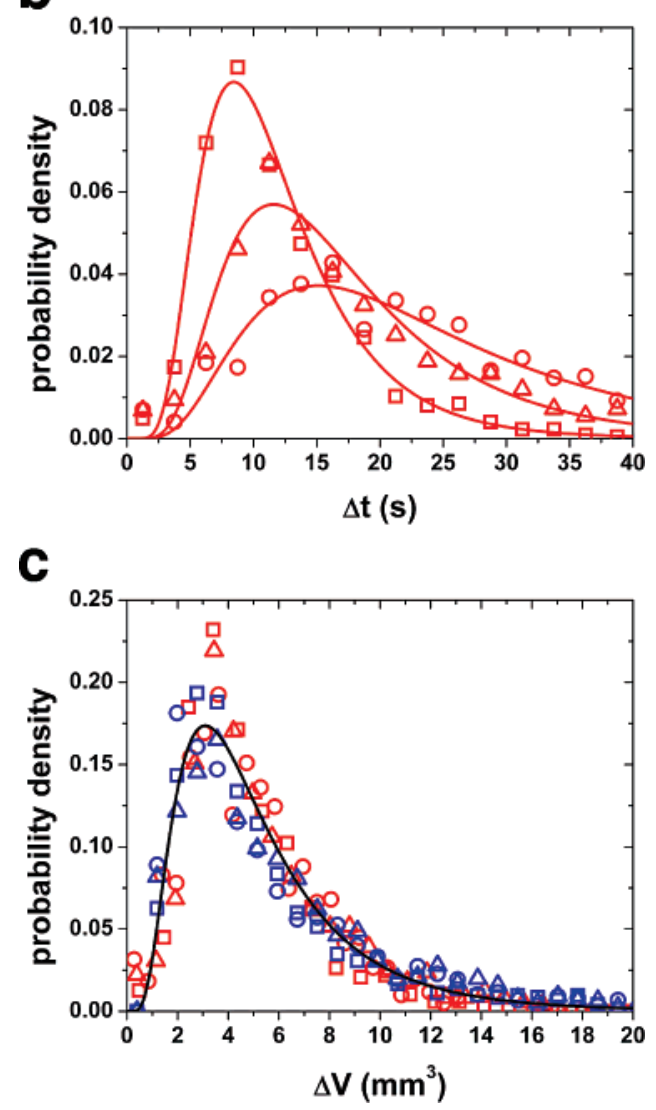

Figure 5. Probability densities characterizing the tube dynamics under fracturing conditions. The variables (a) $\Delta L$ and (b) $\Delta t$ are the length of broken-off tube segments and the time elapsed between subsequent fracturing events, respectively. The normalized distributions of the variable $\Delta V$ (c) are computed from the data in (a) and (b) as $\Delta V=$ $\pi r^{2} \Delta L$ (shown in blue) and $\Delta V=Q \Delta t$ (shown in red), respectively. The solid lines are fitted $\log$-normal distributions. Each plot shows data for three different flow rates: $0.8 \mathrm{~mL} / \mathrm{h}$ (circles), $1.1 \mathrm{~mL} / \mathrm{h}$ (squares), and $1.4 \mathrm{~mL} / \mathrm{h}$ (triangles). Experimental parameters are as follows: $\left[\mathrm{CuSO}_{4}\right]=0.075 \mathrm{M}, \Delta \rho=115 \mathrm{~kg} / \mathrm{m}^{3}$, and $\left[\mathrm{Na}_{2} \mathrm{SiO}_{3}\right]=$ $1.0 \mathrm{M}$.

values correspond to nearly steady growth. The data indicate that the tube can fully detach from the injection nozzle, thus, resetting $L$ to zero. In all experiments on fracturing behavior, the tube length did not exceed $5 \mathrm{~cm}$, which suggests the existence of an upper limit of tube length or indicates that the temporary formation of longer tubes is very unlikely. We emphasize that this feature also distinguishes fracturing growth from all other known regimes because the latter seem to have either no upper length limit or a maximal length that exceeds the height of our solution reservoir (typically $25-30 \mathrm{~cm}$ ). 
TABLE 1: Statistical Data Characterizing Tube Growth in the Fracturing Regime for Three Different Flow Rates $Q$ along with the Average Outer Radius $r$ of the Fractured Tube Segments ${ }^{a}$

\begin{tabular}{cccccccccc}
\hline$Q(\mathrm{~mL} / \mathrm{h})$ & $r(\mathrm{~mm})$ & $\mu_{\Delta L}(\mathrm{~mm})$ & $\sigma_{\Delta L}(\mathrm{~mm})$ & $\mu_{\Delta t}(\mathrm{~s})$ & $\sigma_{\Delta t}(\mathrm{~s})$ & $E(\Delta L)(\mathrm{mm})$ & $\operatorname{var}(\Delta L)(\mathrm{mm})$ & $E(\Delta t)(\mathrm{s})$ & $\operatorname{var}(\Delta t)(\mathrm{s})$ \\
\hline 0.8 & & $2.08 \pm 0.02$ & $0.74 \pm 0.02$ & $3.07 \pm 0.04$ & $0.59 \pm 0.03$ & $10.5 \pm 0.3$ & $80.8 \pm 16.4$ & $25.6 \pm 1.1$ & $274 \pm 88$ \\
1.1 & $0.41 \pm 0.03^{b}$ & $2.21 \pm 0.02$ & $0.72 \pm 0.02$ & $2.73 \pm 0.03$ & $0.53 \pm 0.02$ & $11.8 \pm 0.3$ & $94.8 \pm 19.3$ & $17.6 \pm 0.6$ & $101 \pm 23$ \\
1.4 & & $2.07 \pm 0.01$ & $0.61 \pm 0.01$ & $2.37 \pm 0.01$ & $0.48 \pm 0.01$ & $9.55 \pm 0.11$ & $41.1 \pm 4.3$ & $12.0 \pm 0.1$ & $37.3 \pm 4.3$
\end{tabular}

${ }^{a} \log$ - normal distributions are fitted to the normalized $\Delta L$ and $\Delta t$ distributions yielding the mean values $\left(\mu_{\Delta L}, \mu_{\Delta t}\right)$ and standard deviations $\left(\sigma_{\Delta L}\right.$, $\left.\sigma_{\Delta t}\right)$ of the variables' logarithm. The corresponding expected values are $E(\Delta L)$ and $E(\Delta t)$, and the variances $\operatorname{are}$ denoted as $\operatorname{var}(\Delta t) \operatorname{and} \operatorname{var}(\Delta L)$. ${ }^{b}$ No flow-rate dependence was measured.

Additional information on fracturing dynamics is obtained from statistical analyses. These analyses are based on numerous data sets similar to the ones shown in Figure 4. Each data set spans 15 min of experiment at a typical temporal and spatial resolution of $0.5 \mathrm{~s}$ and $9.3 \mathrm{pixel} / \mathrm{mm}$, respectively. From these data, we extract the probability distributions of the length of the detaching fragments $\Delta L$ and the time passed between subsequent break-off events $\Delta t$. The normalized $\Delta L$ and $\Delta t$ distributions are shown in parts a and b of Figure 5, respectively, for three different flow rates, namely, $0.8 \mathrm{~mL} / \mathrm{h}$ (squares), 1.1 $\mathrm{mL} / \mathrm{h}$ (circles), and $1.4 \mathrm{~mL} / \mathrm{h}$ (triangles). Each distribution has one maximum and approaches zero probability for very small and very large values of $\Delta L$ or $\Delta t$. Moreover, we find that the $\Delta L$ distribution shows either no or very little dependence on the employed flow rate. To the contrary, we measure a pronounced flow-rate dependence for the $\Delta t$ distribution. The maximum of this distribution shifts to larger $\Delta t$ values and also broadens as the flow rate is decreased (see Figure 5b). It is noted that the time elapsed between the $n$th and $(n+1)$ th break-off event shows no strong correlation to the length of the tube fragment released in the $(n+1)$ th event. Correlation analyses of data from 54 different runs at three different flow rates yield correlation coefficients between 0.3 and 0.7 .

The experimental data shown in Figure 5 can be described by $\log -$ normal distributions

$$
f(x)=\frac{1}{x \sigma \sqrt{2 \pi}} \exp \left\{-\frac{(\ln x-\mu)^{2}}{2 \sigma^{2}}\right\}
$$

where $\mu$ and $\sigma$ are the mean and the standard deviation of the variable's logarithm, respectively. The six solid curves in parts $\mathrm{a}$ and $\mathrm{b}$ of Figure 5 represent the corresponding least-square fits and show good agreement with our data. We note that lognormal distributions often apply to measurements that have more or less skewed rather than normal distributions. This situation can arise when mean values are low, variances large, and experimental values are not allowed to be negative. Moreover, $\log$-normal distributions suggest that the measured variable is the multiplicative product of small independent factors. Typical examples are distributions of particles, chemicals, and organisms in the environment, latent periods of infectious diseases, and the length of written sentences. ${ }^{47}$

Table 1 quantifies the main results obtained from fitting $\log -$ normal distributions to the data in parts $\mathrm{a}$ and $\mathrm{b}$ of Figure 5 . It also lists the average outer radius of the broken-off tube segments for three different flow rates. The tube radii are approximately $410 \mu \mathrm{m}$ and appear to be independent of flow rate. The tabulated mean values $\left(\mu_{\Delta L}\right.$ and $\left.\mu_{\Delta t}\right)$ and standard deviations $\left(\sigma_{\Delta L}\right.$ and $\left.\sigma_{\Delta t}\right)$ of the $\Delta L$ and $\Delta t$ distributions re-emphasize that the "fracturing period" decreases with increasing flow rate whereas the length is independent of $Q$. The latter finding implies that the rhythmicity of the process is not simply caused by external, temporal noise such as mechanical or acoustical vibrations of a comparable frequency. To the contrary, the results show that break-off occurs at a characteristic length for which the tube material yields under its own weight. This critical length is best described by the most frequent value of $\Delta L$, which in statistics is called the mode. For log-normal distributions this value equals $\exp \left(\mu_{\Delta L}-\sigma_{\Delta L}^{2}\right)$ and hence yields the critical volume $V_{\text {crit }}=\pi r^{2} \exp \left(\mu_{\Delta L}-\sigma_{\Delta L}^{2}\right)$ which is independent of $Q$. (The physically more relevant critical force is $\Delta \rho g V_{\text {crit }}$ where $g$ denotes earth's acceleration and $\Delta \rho$ is the density difference between the tube and the outer solution.)

Visual inspection of our image data suggests that tubes do not leak during their steady growth phases and hence must be capped at the tip. This observation suggests that the critical volume $V_{\text {crit }}$ can be also described by $V_{\text {crit }}=Q \exp \left(\mu_{\Delta t}-\sigma_{\Delta t}^{2}\right)$. Notice that the latter equation explains why the break-off events shift to longer periods for slower pump rates $Q$. Furthermore, our discussion can be extended to include additional features of the $\Delta L$ and $\Delta t$ distributions. We propose a single master distribution in terms of a variable $\Delta V$ that can be calculated from the $\Delta L$ and $\Delta t$ distributions as $\Delta V=\pi r^{2} \Delta L$ and $\Delta V=$ $Q \Delta t$, respectively. The corresponding, renormalized distributions are shown in Figure 5c. The solid curve represents the best lognormal fit $\left(\mu=(1.51 \pm 0.02) \mathrm{mm}^{3}, \sigma=(0.62 \pm 0.02) \mathrm{mm}^{3}\right)$. All six data sets fall essentially on this master curve, thus implying that all aspects of the $\Delta t$ distributions are determined by the characteristics of the $\Delta L$ distributions.

Last we note that the origins of the observed $\Delta L$ variations are not fully understood. The erratic nature of fracturing dynamics seems to result from variations in the wall's composition, thickness, and diameter. These variations should be characteristic for the employed reactants, but one also needs to consider that the wall is a nonequilibrium material. It is likely that the tensile strength of the wall increases over the lifetime of a given tube segment, thus complicating the overall breakoff dynamics. Also, other factors might have to be considered such as conceivable changes in the tube's internal pressure.

\section{Conclusion}

Our experiments establish that tubular precipitation structures can be created by injection of sodium silicate into a large reservoir containing aqueous cupric sulfate solution. These conditions are the reverse of a situation analyzed in earlier studies where cupric sulfate was injected into a reservoir of sodium silicate solution. Therefore, it is perhaps not surprising that we observed similar growth dynamics in the form of reverse jetting, popping, and budding. However, the tubes formed under reverse conditions are much softer and we did not succeed in collecting tube fragments for space-resolved compositional analyses. The specific layering of materials within the tube wall is expected to be different from that of the materials studied earlier. Pagano et al. reports that tube walls formed by cupric sulfate injection are non-homogeneous materials showing high silica content on the outside and high copper hydroxide content on the inside of the tube. ${ }^{45}$ This layering reflects the geometrical arrangement of the initial reactants, which suggests that tubes grown under reverse conditions should consist predominantly 
of amorphous and/or colloidal silica on the inside and copper hydroxide on the outside.

Reverse reaction conditions also give rise to a growth regime that we coined "fracturing". We reemphasize that fracturing has not been observed under "non-reverse" conditions. Furthermore, it differs qualitatively from the other three regimes and is periodic although the time elapsed between subsequent breakoff events fluctuates greatly. We are confident that this erratic behavior is not caused by external factors but rather reflects spatial variations of the tube material. This conclusion is drawn from (i) the observation of a flow-rate-independent $\Delta L$ distribution and (ii) the existence of a master curve onto which all $\Delta L$ and $\Delta t$ data can be scaled. In the future, it will be interesting to study fracturing dynamics for other reactant pairs and perhaps also for externally forced systems. Such experiments should provide more insights into the specifics of fracturing dynamics and will expand our overall understanding of precipitation tubes in artificial and natural systems.

Acknowledgment. Acknowledgement is made to the Donors of the American Chemical Society Petroleum Research Fund for support of this research. We thank Th. M. Fischer for discussions.

\section{References and Notes}

(1) Double, D. D.; Hellawell, A.; Perry, S. J. Proc. R. Soc. London, Ser. A 1978, 359, 435 .

(2) Sørensen, T. S. J. Colloid Interface Sci. 1981, 79, 192.

(3) Double, D. D. Philos. Trans. R. Soc. London, Ser. A 1983, 310 , 55.

(4) Mann, S. Biomineralization: Principles and Concepts in Bioinorganic Materials Chemistry; Oxford University Press: Oxford, U.K., 2001.

(5) Garciá-Ruiz, J. M.; Hyde, S. T.; Carnerup, A. M.; Christy, A. G.;

Van Kranendonk, M. J.; Welham, N. J. Science 2003, 302, 1194.

(6) Ackermann, A. Colloid Z. 1921, 28, 270.

(7) Ackermann, A. Colloid Z. 1932, 59, 49.

(8) Fontana, M. G. Corrosion Engineering; McGraw-Hill: New York, 1986.

(9) Coatman, D.; Thomas, N. L.; Double, D. D. J. Mater. Sci. 1980, 15, 2017.

(10) Stone, D. A.; Goldstein, R. E. Proc. Natl. Acad. Sci. U.S.A. 2004, 101, 11537.

(11) Stone, D. A.; Lewellyn, B.; Baygents, J. C.; Goldstein, R. E. Langmuir 2005, 21, 10916.

(12) Curl, R. L. NSS Bull. 1972, 34, 129-136.

(13) White, W. B. In Cave Minerals and Spelotherms. The Science of Speology; T. D. Ford, T. D., Cullingford, C. H. D., Eds.; Academic Press: London, 1976; p 267.

(14) Short, M. B.; Baygents, J. C.; Goldstein, R. E. Phys. Fluids 2005, $17,083101$.

(15) Short, M. B.; Baygents, J. C.; Beck, J. W.; Stone, D. A.; Toomey, R. S.; Goldstein, R. E. Phys. Rev. Lett. 2005, 94, 018501.
(16) Corliss, J. B.; Dymond, J.; Gordon, L. I.; Edmond, J. M.; Von Herzen, R. P.; Ballard, R. D.; Green, K.; Williams, D.; Bainbridge, A.; Crane, K.; van Andel, Tj. H. Science 1979, 293, 1073-1083.

(17) Maselko, J.; Strizhak, P. J. Phys. Chem. B 2004, 108, 4937.

(18) Dent Glasser, L. S. Chem. Br. 1982, 18, 33. 145.

(19) Collins, C.; Zhou, W.; Klinowski, J. Chem. Phys. Lett. 1999, 306 ,

(20) Balköse, D.; Özkan, F.; Köktürk, U.; Ulutan, S.; Ülkü, S.; Nişli, G. J. Sol-Gel Sci Technol. 2002, 23, 253.

(21) Maselko, J.; Geldenhuys, A.; Miller, J.; Atwood, D. Chem. Phys. Lett. 2003, 373, 563 .

(22) Bormashenko, E.; Bormashenko, Y.; Stanevsky, O.; Pogreb, R. Chem. Phys. Lett. 2006, 417, 341.

(23) Bormashenko, E.; Bormashenko, Y.; Stanevsky, O.; Pogreb, R.; Whyman, G.; Stein, T.; Barkay, Z. Colloids Surf., A 2006, 289, 245.

(24) Damerell, V. R.; Brock, H. J. Chem. Educ. 1949, 26, 148.

(25) Webster, M. Educ. Chem. 1998, 35, 126.

(26) Glauber, J. R. Furni Novi Philiosophici; Amsterdam, The Netherlands, 1646 .

(27) Keir, J. Philos. Trans. R. Soc. London 1790, 80, 359

(28) Leduc, S. The Mechanism of Life; Rebman: London, 1911.

(29) Roesky, H. W.; Möckel, K. Chemical Curiosities; VCH: Weinheim, Germany, 1996.

(30) Mann, T. Doktor Faustus; Bermann-Fischer: Stockholm, Sweden, 1947.

(31) Sacks, O. Uncle Tungsten: Memories of a Chemical Boyhood; Vintage: New York, 2001.

(32) Collins, C.; Zhou, W.; Mackay, A. L.; Klinowski, J. Chem. Phys. Lett. 1998, 286, 88.

(33) Collins, C.; Mann, G.; Hoppe, E.; Duggal, T.; Barr, T. L.; Klinowski, J. Phys. Chem. Chem. Phys. 1999, 1, 3685.

(34) Collins, C.; Makoya, R.; Klinowski, J. Phys. Chem. Chem. Phys. 1999, 1, 4669.

(35) Duan, W.; Kitamura, S.; Uechi, I.; Katsuki, A.; Tanimoto, Y. J. Phys. Chem. B 2005, 109, 13445.

(36) Jones, D. E. H.; Walter, U. J. Colloid Interface Sci. 1998, 203, 286.

(37) Jones, D. E. H. Am. Sci. 2002, 90, 454.

(38) Stockwell, B.; Williams, A. Sch. Sci. Rev. 1994, 76, 7.

(39) Cartwright, J. H. E.; Garciá-Ruiz, J. M.; Novella, M. L.; Otálora, F. J. Colloid Interface Sci. 2002, 256, 351.

(40) Preece, S. J.; Billingham, J.; King, A. C. J. Eng. Math. 2001, 40, 43-58.

(41) Hazelhurst, T. H. J. Chem. Educ. 1941, 18, 286.

(42) Thouvenel-Romans, S.; Steinbock, O. J. Am. Chem. Soc. 2003, 125, 4338.

(43) Thouvenel-Romans, S.; van Saarloos, W.; Steinbock, O. Europhys. Lett. 2004, 67, 42.

(44) Thouvenel-Romans, S.; Pagano, J. J.; Steinbock, O. Phys. Chem. Chem. Phys. 2005, 7, 2610.

(45) Pagano, J. J.; Thouvenel-Romans, S.; Steinbock, O. Phys. Chem. Chem. Phys. 2007, 9, 110-118.

(46) Söhnel, O.; Novotný, P. Density of Aqueous Solutions of Inorganic Substances; Elsevier: Amsterdam, The Netherlands, 1985.

(47) Limpert, E.; Stahel, W. A.; Abbt, M. Bio. Sci. 2001, 51, 341- 